\title{
NOTIZEN
}

Bildung und Reaktion

\section{Si-fluorierter 1.3.5-Trisila-cyclohexane}

Formation and Reactions

of Si-fluorinated 1,3,5-Trisilacyclohexanes

\section{G. Fritz und M. Portner}

Institut für Anorganische Chemie der Universität Karlsruhe

(Z. Naturforsch. 30b, 965-968 [1975]; eingegangen am 11. Juni 1975)

Si-fluorinated 1,3,5-Trisilacyclohexane

The reaction of 1,3,5-Trisilacyclohexanes containing $\mathrm{Si}-\mathrm{F}$ and $\mathrm{C}-\mathrm{Cl}$ groups and $\mathrm{CH}_{3} \mathrm{MgCl}$ starts with methylation of the $\mathrm{Si}-\mathrm{F}$ group (in contrast to the reactions of the corresponding $\mathrm{Si}-\mathrm{Cl}$ containing derivatives). At a certain degree of methylation a ring contraction starts at the $\mathrm{C}-\mathrm{Cl}$ group.

Aus vorausgehenden Untersuchungen ist bekannt, daß $\left(\mathrm{Cl}_{2} \mathrm{Si}-\mathrm{CCl}_{2}\right)_{3}$ mit $\mathrm{meMgCl}\left(\mathrm{me}=\mathrm{CH}_{3}\right)$ unter Ringverengung zu 1.1-Dichlor-3.3-dimethyl-4-trimethylsilyl-5-monochlor-1.3-disila-cyclopenten reagiert $^{1}$, das sich mit weiterem meMgCl unter Ringspaltung zum $\mathrm{me}_{3} \mathrm{Si}-\mathrm{CCl}_{2}-\mathrm{Sime}_{2}-\mathrm{C} \equiv \mathrm{C}-\mathrm{Sime}_{3}$ umsetzt $^{2}$. Die Reaktion beginnt an der CCl-Gruppe des $\left(\mathrm{Cl}_{2} \mathrm{Si}-\mathrm{CCl}_{2}\right)_{3}$. Enthält das Si-chlorierte 1.3.5Trisila-cyclohexan eine $\mathrm{CCl}_{2}$-Gruppe, so wird diese mit $\mathrm{meMgCl}$ in eine Vinylgruppe überführt ${ }^{1}$. Die Bildung von C-chlorierten, Si-methylierten Derivaten des 1.3.5-Trisila-cyclohexans, wie zum Beispiel das $\left(\mathrm{me}_{2} \mathrm{Si}-\mathrm{CCl}_{2}\right)_{3}$, ist auf diesem Wege nicht möglich. Derartige Verbindungen sind für die Synthese multicyclischer Carbosilane von Interesse. Da von der Polarität der Si-Halogenbindung ein Einfluß auf die $\mathrm{CCl}_{2}$-Gruppe $\mathrm{zu}$ erwarten ist, wurde die Synthese der entsprechenden SiF-haltigen Derivate angestrebt. Ihre Bildung wurde nach (1) und (2) ermöglicht.

Verbindung 1 läßt sich mit meMgCl methylieren. So führt die Umsetzung mit $3 \mathrm{Mol}$ meMgCl in $\left(\mathrm{C}_{2} \mathrm{H}_{5}\right)_{2} \mathrm{O}$ (Reaktionsbeginn bei $-70^{\circ} \mathrm{C}$, langsam erwärmen auf $20^{\circ} \mathrm{C}$ ) bevorzugt zum (meFSi-CH $\left.{ }_{2}\right)_{3}$, das sich mit $\mathrm{LiAlH}_{4}$ in $\left(\mathrm{meHSi}-\mathrm{CH}_{2}\right)_{3}$ überführen läßt.

Verbindung 2 reagiert mit meMgCl unter Substitution der SiF-Gruppen. Mit einem Überschuß an meMgCl entsteht als Hauptprodukt nach (3) Verbindung $\mathbf{5}$.

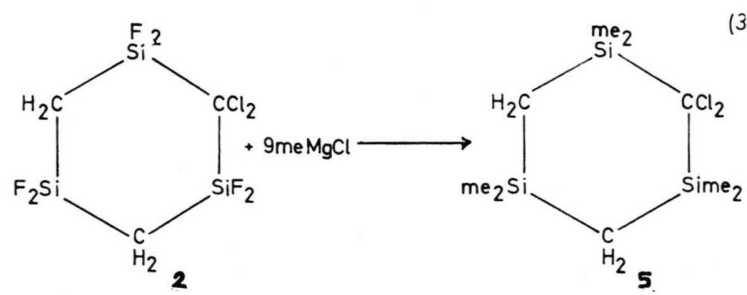

Sonderdruckanforderungen an Prof. Dr. G. FRITZ, D-7500 Karlsruhe 1, Englerstraße 11, Institut für Anorganische Chemie der Universität, BRD.

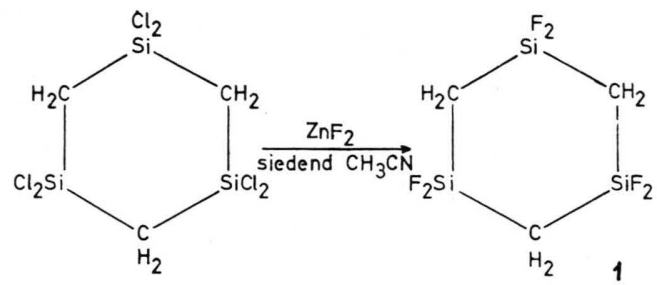
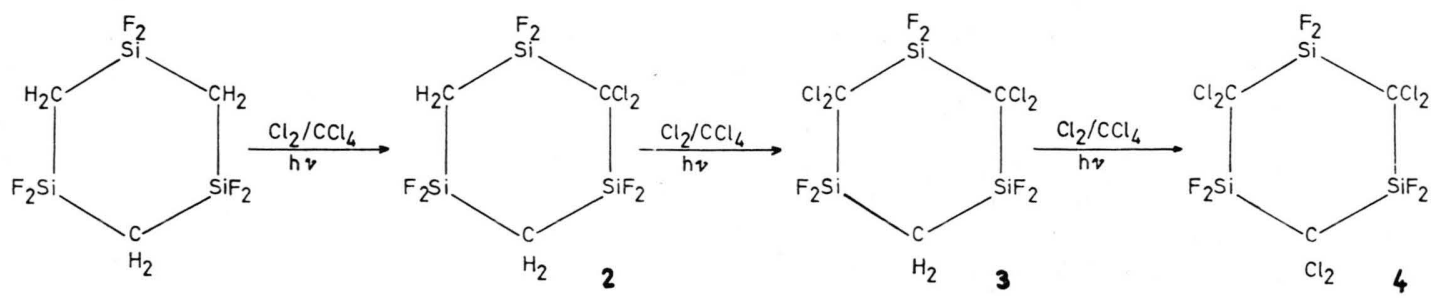

(2) 
Mit $3 \mathrm{Mol} \mathrm{meMgCl}$ bildet sich aus 2 Verbindung $\mathbf{6}$, die mit $\mathrm{LiAlH}_{4}$ in Verbindung 7 überführt werden kann.<smiles>[1H][Si]1(F)C[Si](F)(F)C[Si](F)(F)C1</smiles>

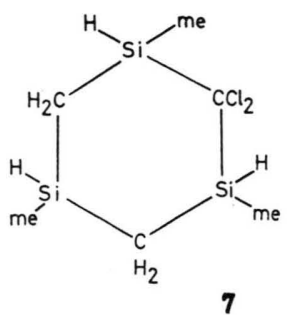

Auch Verbindung 3 läßt sich Si-methylieren. So bildet sich nach (4) in 70-proz. Ausbeute Verbindung 8 mit zwei $\mathrm{CCl}_{2}$-Gruppen neben geringen $\mathrm{An}$ teilen von Verbindungen, die durch Spaltung des Sechsringes entstehen.

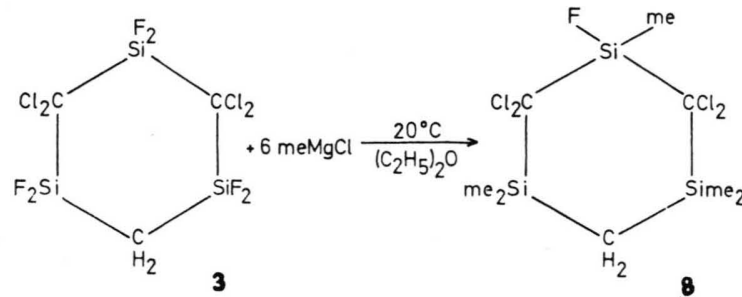

Die Spaltung des Sechsringes wird durch polare Lösungsmittel bzw. durch Umsetzung mit $\mathrm{LiCH}_{3}$ begünstigt. So wird Verbindung 3 mit $6 \mathrm{Mol} \mathrm{meMgCl}$ in THF bei $-78^{\circ} \mathrm{C}$ (Reaktionsbeginn, anschließend erwärmen auf $20^{\circ} \mathrm{C}$ ) vollständig gespalten und die Reaktion mit $6 \mathrm{Mol} \mathrm{LiCH}_{3}$ bei $-78{ }^{\circ} \mathrm{C}$ in $\left(\mathrm{C}_{2} \mathrm{H}_{5}\right)_{2} \mathrm{O}$ führt ebenfalls zur vollständigen Spaltung des Ringes.

Einen Einblick in die Spaltungsreaktion vermittelt die Umsetzung von Verbindung $8 \mathrm{mit}$ einem Überschuß an meMgCl nach (5).
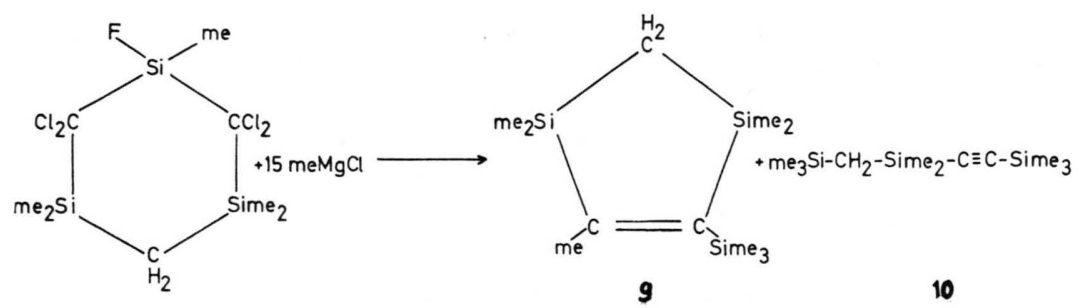

Mit $\mathrm{LiCH}_{3}$ (Überschuß) erfolgt nahezu quantitativ die Spaltung zu me $3 \mathrm{Si}-\mathrm{CH}_{2}-\mathrm{Sime}_{2}-\mathrm{C} \equiv \mathrm{C}-\mathrm{Sime}_{3}$. Auch Verbindung 4 läßt sich nach (6) methylieren, wobei Verbindung 11 mit 85-proz. Ausbeute isoliert wurde.

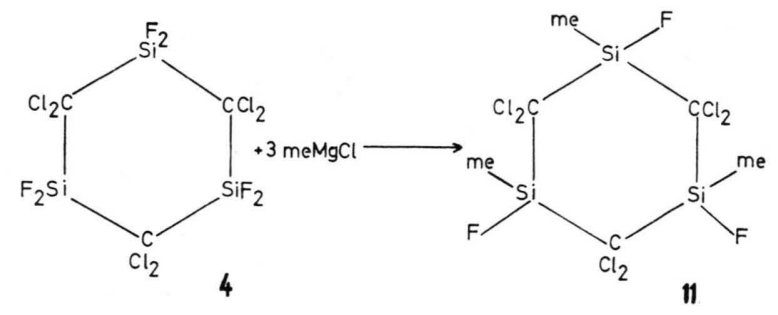

(6)

Die weitere Umsetzung von 11 mit meMgCl oder $\mathrm{LiCH}_{3}$ führt zur Aufspaltung des Ringgerüstes von 11.

Aus den Ergebnissen ist zu erkennen, daß bei den $\mathrm{SiF} / \mathrm{CCl}$-haltigen 1.3.5-Trisilacyclohexanen die Reaktion mit einer normalen SiF-Methylierung beginnt. Wenn ein bestimmter Methylierungsgrad erreicht ist, setzt die Reaktion an der CCl-Gruppe ein und führt nach dem bei den SiCl-haltigen Derivaten bekannten Mechanismus ${ }^{1}$ zur Ringverengung. Für Verbindung 3 ergibt sich somit Reaktionsweg (7):

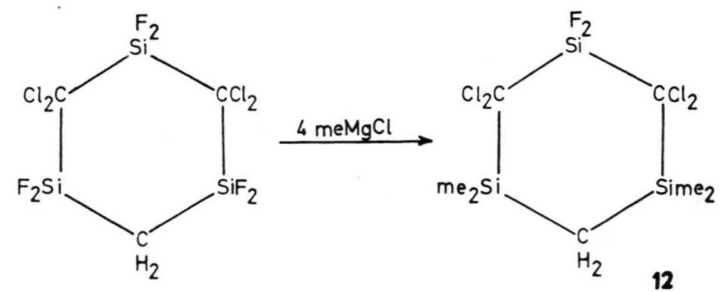


Mit Verbindung 12 ist die Methylierungsstufe erreicht, bei der die Reaktion an der CCl-Gruppe nach (8) einsetzt:

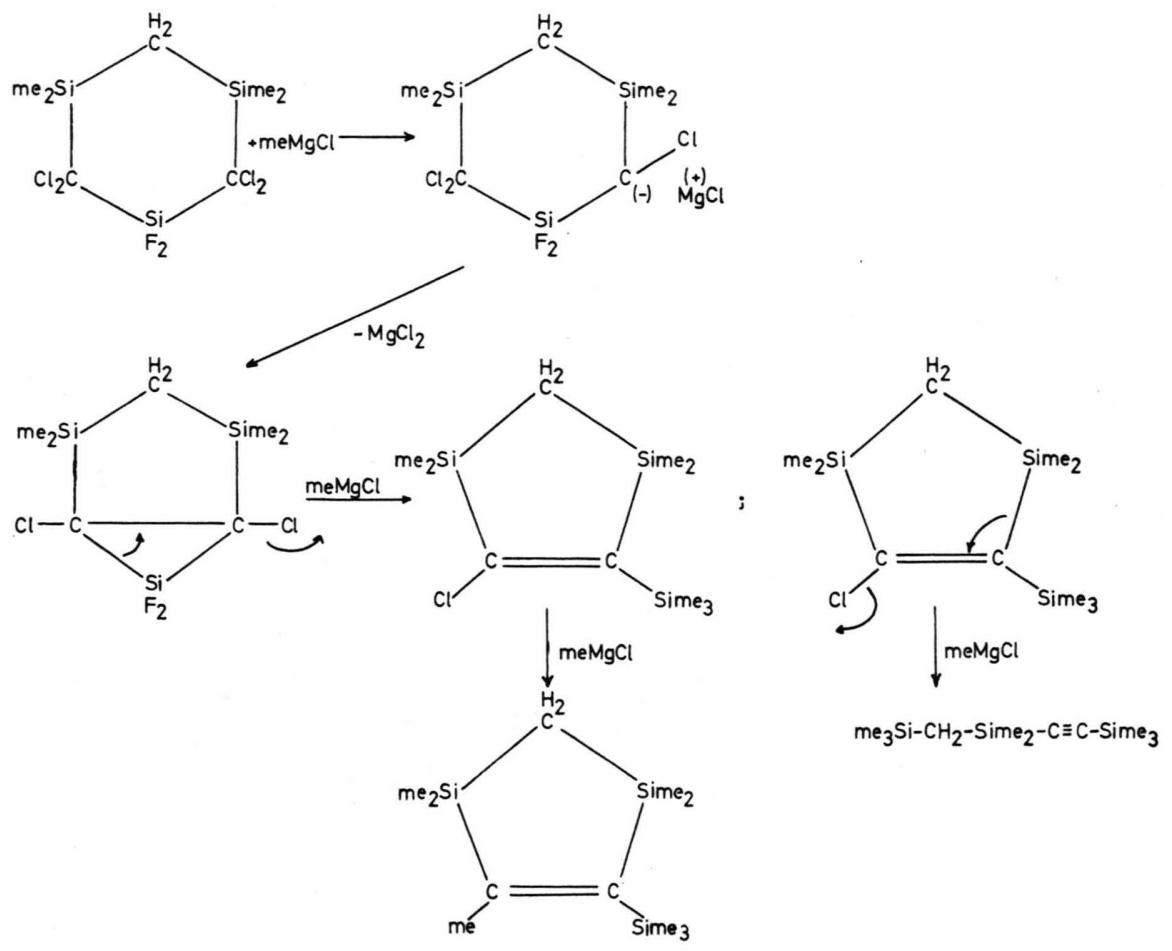

(8)

Offenbar wird durch die Teilmethylierung an den Si-Atomen die Reaktionsfähigkeit der CClGruppe so begünstigt, daß nun von ihr ausgehend die Ringverengungsreaktion einsetzen kann. Die angegebenen Verbindungen wurden durch chemische Analyse, NMR-Untersuchung und massenspektrometrische Untersuchung gesichert.

Tab. I. Analysendaten der Verbindungen.

\begin{tabular}{|c|c|c|c|c|c|c|c|c|c|c|}
\hline \multirow[b]{2}{*}{ Verbindung } & \multirow[b]{2}{*}{ MG } & \multicolumn{3}{|c|}{ berechnet } & \multirow[b]{2}{*}{ MG } & \multicolumn{3}{|c|}{ gefunden } & \multirow{2}{*}{\multicolumn{2}{|c|}{$\begin{array}{l}\text { Schmp. } \\
{\left[{ }^{\circ} \mathrm{C}\right]}\end{array}$}} \\
\hline & & $\begin{array}{l}\mathrm{Si} \\
{[\%]}\end{array}$ & $\begin{array}{l}\mathrm{Cl} \\
{[\%]}\end{array}$ & $\begin{array}{l}\mathrm{F} \\
{[\%]}\end{array}$ & & $\begin{array}{l}\mathrm{Si} \\
{[\%]}\end{array}$ & $\begin{array}{l}\mathrm{Cl} \\
{[\%]}\end{array}$ & $\begin{array}{l}\mathbf{F} \\
{[\%]}\end{array}$ & & \\
\hline 1 & 240,33 & 35,06 & - & 47,43 & 243,5 & 36,9 & 一 & 48,1 & & $141-143$ \\
\hline 2 & 309,22 & 27,25 & 22,93 & 36,86 & 306,1 & 28,2 & 23,7 & 35,6 & & $56-58$ \\
\hline 3 & 378,11 & 22,28 & 37,52 & 30,15 & 381,3 & 23,4 & 38,8 & 33,7 & & $45-47$ \\
\hline 4 & 447,00 & 18,85 & 47,59 & 25,50 & 449,8 & 17,0 & 46,5 & 26,9 & & $40-42$ \\
\hline 6 & 297,33 & 28,34 & 23,85 & 19,17 & 280,6 & 25,1 & 25,3 & 21,8 & $a b$ & 93 \\
\hline 8 & 358,29 & 23,52 & 39,58 & 5,30 & 364,1 & 22,2 & 41,4 & 5,0 & & $118-120$ \\
\hline 11 & 435,11 & 19,36 & 48,89 & 13,10 & 433,3 & 19,1 & 48,6 & 12,2 & $a b$ & 189,5 \\
\hline 12 & 362,26 & 23,26 & 39,15 & 10,49 & 352,0 & 21,7 & 40,1 & 7,0 & & $123-126$ \\
\hline
\end{tabular}

Die Verbindungen 6, 7, 8, 11 liegen als nicht getrennte Konformationsisomere vor. Die Verbindungen 5 u. 7 sind flüssig und wurden durch fraktio- nierte Kondensation zwischen $25-35^{\circ} \mathrm{C} / 10^{-3}$ Torr abgetrennt. 
Die Verbindungen 5, 7, 9 und $\mathbf{1 0}$ wurden durch massenspektrometrische Untersuchung gesichert.

\begin{tabular}{|c|c|c|}
\hline & Masse & $\begin{array}{l}{ }^{28} \mathrm{SiC}_{8} \mathrm{H}_{19}{ }^{35} \mathrm{Cl}_{2} \\
\text { ber. } 269,017170 \\
\text { gef. } 269,017853\end{array}$ \\
\hline 7 & " & $\begin{array}{l}{ }^{28} \mathrm{Si}_{3} \mathrm{C}_{5} \mathrm{H}_{13}{ }^{35} \mathrm{Cl}_{2}\left(\mathrm{M}-\mathrm{CH}_{3}\right) \\
\text { ber. } 226,970220 \\
\text { gef. } 226,970692 \\
{ }_{28} \mathrm{Si}_{3} \mathrm{C}_{5} \mathrm{H}_{13}{ }^{35} \mathrm{Cl}^{37} \mathrm{Cl}\left(\mathrm{M}-\mathrm{CH}_{3}\right) \\
\text { ber. } 228,967269 \\
\text { gef. } 228,967547\end{array}$ \\
\hline 9 & , & $\begin{array}{l}{ }^{28} \mathrm{Si}_{3} \mathrm{C}_{11} \mathrm{H}_{26}\left(\mathrm{M}-\mathrm{CH}_{3}\right) \\
\text { ber. } 242,134237 \\
\text { gef. } 242,133866\end{array}$ \\
\hline 10 & , & 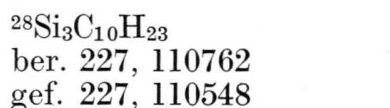 \\
\hline
\end{tabular}

Die Umsetzungen der $\mathrm{CCl}_{2}$-haltigen 1.3.5-Trisilacyclohexane mit einem Überschuß an meMgCl werden zweckmäßig bei niedriger Temp. durchgeführt, z.B. (3), um Ringspaltungen zu vermeiden.

Zur Umsetzung von Verbindung 2 wurden $4,6 \mathrm{~g}$ von 2 in $200 \mathrm{ml}$ Diäthyläther $\left(\mathrm{Et}_{2} \mathrm{O}\right)$ vorgelegt, auf $-78{ }^{\circ} \mathrm{C}$ gekühlt und die meMgCl-Lösung (herge-

1 G. Fritz u. M. Szczepanski, Z. Anorg. Allg. Chem. 367, 44 [1969]; G. Fritz u. N. Braunagel, Z. Anorg. Allg. Chem. 399, 280 [1973]. stellt aus $3,9 \mathrm{~g} \mathrm{Mg}$ in $390 \mathrm{ml} \mathrm{Et}_{2} \mathrm{O}$; Molverhältnis 1:9) innerhalb von 2 Stdn. zugetropft. Anschließend wurde das Reaktionsgemisch unter Rühren innerhalb 24-28 Stdn. auf $20^{\circ} \mathrm{C}$ erwärmt. Zur Aufarbeitung wurde das Lösungsmittel abkondensiert, der Rückstand in Pentan aufgenommen, die Mg-Salze abfiltriert, das Pentan abdestilliert und $3,3 \mathrm{~g}$ flüssige Reaktionsprodukte erhalten. Bei der anschließenden fraktionierten Kondensation $\left(25^{\circ} \mathrm{C} /\right.$ $10^{-4}$ Torr) wurden 2,3 g Substanz erhalten, die zu $70 \%$ aus Verbindung $\mathbf{5}$ bestehen; Rückstand $0,9 \mathrm{~g}$.

Zur Bildung der Verbindung 8 nach (4) wurden $5 \mathrm{~g}$ von Verbindung 3 (gelöst in $140 \mathrm{ml} \mathrm{Et}{ }_{2} \mathrm{O}$ ) vorgelegt, $6 \mathrm{Mol}$ meMgCl [dargestellt aus 2, $11 \mathrm{~g} \mathrm{Mg}$ $\left(10 \%\right.$ Überschuß); pro g Mg $\left.100 \mathrm{ml} \mathrm{Et}{ }_{2} \mathrm{O}\right]$ innerhalb $1,5 \mathrm{Stdn}$. bei $20{ }^{\circ} \mathrm{C}$ zugetropft und das Reaktionsgemisch dann noch 24 Stdn. bei $20^{\circ} \mathrm{C}$ gerührt. Anschließend wurde das Lösungsmittel bei Unterdruck abkondensiert (Vorlage gekühlt mit fl. $\mathrm{N}_{2}$ ) und aus dem dann anfallenden Rohprodukt die Verbindung 8 bei $45-50^{\circ} \mathrm{C} / 2,5 \quad 10^{-3}$ Torr absublmiert. Isoliert wurden $2,8 \mathrm{~g}$ von $8(60 \%$ bezogen auf die eingesetzte Verbindung 3), wobei der Rückstand von etwa $0,9 \mathrm{~g}$ noch Verbindung 8 enthielt.

Herrn Dr. Scheer danken wir für die Durchführung der massenspektrometrischen Untersuchung.

2 G. Fritz u. P. Böttinger, Z. Anorg. Allg. Chem. 395,159 [1973]. 